ISSN 0103-5150

Fisioter. Mov., Curitiba, v. 26, n. 2, p. página 315-320, abr./jun. 2013

Licenciado sob uma Licença Creative Commons

\title{
Relação entre depressão e desequilíbrio postural em idosos que sofreram acidente vascular encefálico
}

\author{
Relationship between depression and postural imbalance \\ in the elderly who have suffered stroke
}

\author{
Ana Flávia Dias Tanaka ${ }^{[a]}$, Marcos Eduardo Scheicher ${ }^{[b]}$ \\ [a] Terapeuta Ocupacional graduada pela Universidade Estadual Paulista (Unesp), Marília, SP - Brasil, e-mail: \\ aftanaka@hotmail.com \\ [b] Doutor pela Faculdade de Medicina da Universidade de São Paulo (USP- Ribeirão Preto), professor do curso \\ de Fisioterapia da Universidade Estadual Paulista (Unesp), professor do Programa de Pós-Graduação em \\ Desenvolvimento Humano e Tecnologias da Universidade Estadual Paulista (Unesp), Rio Claro, SP - Brasil, e-mail: \\ mscheicher@marilia.unesp.br
}

\section{Resumo}

Introdução: A incidência de Acidente Vascular Encefálico (AVE) aumenta com o envelhecimento e duplica a cada década de vida a partir dos 55 anos de idade. Entre as complicações decorrentes de um AVE estão o desequilíbrio postural e a depressão. Objetivos: Avaliar e correlacionar equilíbrio postural e depressão em idosos com e sem AVE. Materiais e métodos: Foram avaliados 38 sujeitos (19 com AVE e 19 sem AVE). 0 equilíbrio foi avaliado pela Escala de Equilíbrio de Berg e a depressão pela Escala de Depressão de Yesavage. Resultados: Idosos com AVE apresentaram depressão quando comparados com idosos sem a doença $(\mathrm{p}<0,0001)$. 0 risco de cair em idosos com AVE foi 102 vezes maior do que em idosos sem AVE. Houve correlação entre depressão e equilíbrio $(r=-0,55 ; p=0,01)$. Conclusão: Pacientes idosos com sequelas de AVE apresentam maior desequilíbrio e maior depressão, quando comparados com idosos de mesma faixa etária sem a doença.

Palavras-chave: Acidente Cerebral Vascular. Quedas. Depressão.

\section{Abstract}

Introduction: The incidence of stroke it increases with the aging and each successive decade above 55 years leads to a doubling of stroke incidence. Among the current complications of stroke are the unbalance postural 
and depression. Objectives: To evaluate and correlate the postural balance and depression in elderly with and without stroke. Materials and methods: We evaluated 38 subjects (19 with stroke and 19 without). The balance was evaluated by Berg Balance Scale and the depression by Yesavage Depression Scale. Results: Elderly with stroke presented depression when compared with elderly without the disease $(p<0.0001)$. The risk of falling in elderly with stroke was 102 times higher than in elderly without stroke. There was a correlation between depression and balance ( $r=-0.55 ; p=0.01)$. Conclusion: Elderly patients with sequels of stroke have unbalance and greater depression, when compared with elderly of same age without the disease.

Keywords: Stroke. Falls. Depression.

\section{Introdução}

A incidência de Acidente Vascular Encefálico (AVE) aumenta com o envelhecimento (1) e duplica a cada década de vida a partir dos 55 anos de idade (2). A combinação do aumento da expectativa de vida e dos avanços na área médica tem resultado em um grande número de idosos que sobrevivem após um AVE (3). A prevalência de morbidade em pessoas pós-AVE varia entre $24 \%$ e $54 \%$ (4). Entre os problemas que podem atingir uma pessoa com AVE estão as sequelas físicas e psicológicas, as quais podem ser devastadoras (5).

Alterações decorrentes do AVE influenciam os elementos do controle postural, resultando em falhas no processo de integração sensorial e geração de respostas motoras. Aproximadamente $40 \%$ das pessoas caem no primeiro ano após ter sofrido o AVE $(6,7)$ e durante os 30 meses seguintes à alta hospitalar, as quedas tornam-se episódios comuns para elas (6). Existem várias razões para que uma pessoa após um AVE tenha dificuldade de manter o equilíbrio corporal: fraqueza muscular, desempenho motor prejudicado e alteração do tônus muscular, redução do movimento articular, dor e dificuldades com a visão. Após um AVE, observa-se aumento no risco de ocorrência de quedas, o que pode acarretar medo de cair (8) e depressão (9).

A depressão tem sido considerada a consequência neuropsiquiátrica mais comum após a ocorrência de um AVE (10), estimando-se que $1 / 3$ desses pacientes desenvolvem um quadro depressivo (11); essas pessoas podem ter mais prejuízos na realização de atividades de vida diária (AVD), necessitando de maiores cuidados.

Além disso, existem outros problemas que pacientes com depressão pós-AVE podem desenvolver: diminuição da função cognitiva, da velocidade de recuperação e das habilidades sociais, e morte $(12,13)$.

Diante disso, os objetivos deste estudo foram: avaliar e comparar o equilíbrio e a presença de depressão em idosos com e sem diagnóstico de AVE e correlacionar a depressão com os escores da escala de equilíbrio.

\section{Casuística e métodos}

Foram avaliados 38 participantes separados em 2 grupos: grupo 1 - 19 idosos com história de AVE; grupo 2 - 19 idosos sem história, de acordo com os seguintes critérios: ter, no momento da pesquisa, idade igual ou superior a 60 anos, ter sofrido AVE (para o grupo 1), ter a pontuação do Mini Exame do Estado Mental (MEEM) compatível com a escolaridade (18), não ser cadeirante e não apresentar alterações fisiológicas graves. 0 MEEM é uma ferramenta que tem sido usada em ambientes clínicos e em pesquisa com o objetivo de rastreio de quadros demenciais e detecção de declínio cognitivo.

O projeto foi submetido ao Comitê de Ética em Pesquisa da Faculdade de Medicina de Marília (Famema) e aprovado com o protocolo número $144 / 09$. Todos os participantes da pesquisa assinaram um Termo de Consentimento Livre e Esclarecido.

\section{Avaliação do risco de quedas}

0 equilíbrio postural foi avaliado por meio da Escala de Equilíbrio de Berg (EEB) - versão brasileira $(14,15,16,17)-$-, composta por 14 questões; as pontuações dos subitens variam de 0 a 4 pontos (0 - incapaz de realizar e 4 - realiza com independência). 0 teste avalia a forma como é realizado cada item 
e o tempo em que este se realiza. Os escores totais variam de 0 a 56 pontos, sendo que a máxima pontuação está relacionada a um melhor desempenho. Escores abaixo de 45 indicam grande risco de quedas.

\section{Avaliação da Depressão}

A depressão foi avaliada pela Escala de Depressão Geriátrica em versão reduzida de Yesavage (GDS-15). Trata-se de um teste com 15 perguntas negativo-afirmativas, sendo contado 1 ponto para cada resposta do paciente que corresponder à resposta em negrito. Os pontos de corte utilizados foram: ( 0 a 5 ) ausência de depressão, (6 a 10) depressão moderada e (11 a 15) depressão grave. Almeida e Almeida (17) demonstraram que a versão brasileira da GDS oferece medidas válidas para o diagnóstico de depressão entre os idosos.

\section{Análise estatística}

Os dados foram apresentados como média \pm desvio padrão. Após verificação da normalidade e homogeneidade dos dados, foram adotados testes estatísticos apropriados para a análise das variáveis entre os grupos. Dessa forma, as comparações dos escores da Escala de Equilíbrio de Berg e da Escala Geriátrica de Depressão entre os grupos foram feitas por meio do teste de Mann-Whitney e as correlações entre a Escala Geriátrica de Depressão e a Escala de Equilíbrio de Berg foram feitas por meio do índice de correlação de Spearman. 0 risco de quedas foi avaliado por meio do Odds Ratio, considerando um escore $\leq$ 45 (18), para maior risco de quedas. Foi aceito como significante um valor de $\mathrm{p} \leq 0,05$.

\section{Resultados}

Participaram do estudo 19 idosos com AVE e 19 sem AVE, não havendo diferença significativa entre as idades dos participantes $(67,3 \pm 5,9$ e 66,1 $\pm 5,7$, respectivamente). Foi observada diferença significativa $(\mathrm{p}<0,0001)$ entre os valores do MEEM para o grupo AVE $(25,3 \pm 2,7)$ e para o grupo sem AVE $(28,6 \pm 1,8)$; porém, como os dois grupos ficaram acima do valor estipulado para alterações cognitivas ( $\geq 18$ pontos), esse resultado não tem significância clínica para o estudo.
Comparação do risco de quedas

O Gráfico 1 mostra o número de idosos com e sem AVE distribuídos nos grupos de maior e menor risco de quedas. Percebe-se que 14 dos 19 idosos com AVE $(73,68 \%)$ estão no grupo de maior risco de quedas e $5(26,38 \%)$ no grupo de menor risco; dos idosos sem AVE, 19 (100\%) estão no grupo de menor risco de quedas. A Odds Ratio mostrou que idosos com AVE apresentam 102 vezes mais risco de cair do que idosos sem AVE $(p<0,0001)$.

\section{Comparação da presença de depressão}

O Gráfico 2 mostra a comparação dos escores da escala de depressão para idosos com e sem AVE. Observa-se que no grupo com AVE há ocorrência de depressão, enquanto que no grupo sem AVE, essa situação não ocorre $(\mathrm{p}<0,0001)$.

Relação entre depressão e risco de quedas

O Gráfico 3 mostra correlação entre os escores da Escala de Depressão Geriátrica e os valores da Escala de Equilíbrio de Berg para idosos com AVE. Observa-se uma tendência moderada de que, quanto mais depressivo, maior a probabilidade de quedas $(r=-0,55 ; p=0,01)$. No grupo sem AVE, não foram encontrados escores que indicassem depressão.

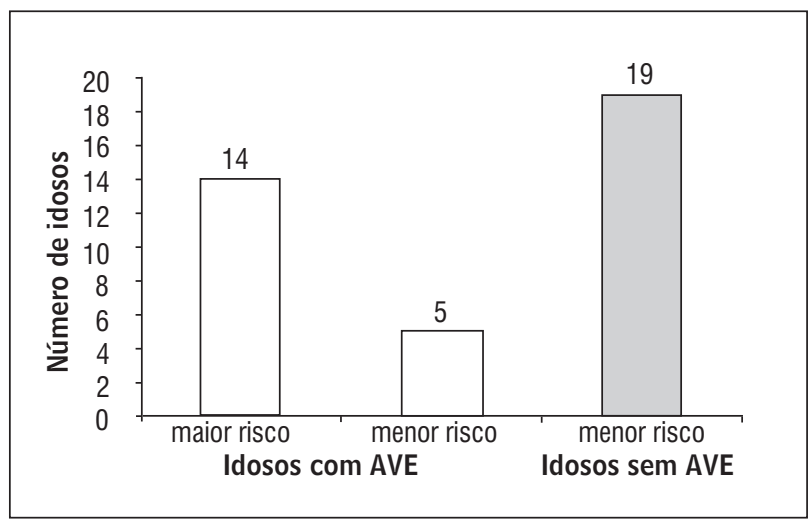

Gráfico 1 - Distribuição de idosos com maior e menor risco de quedas, de acordo com a Escala de Equilíbrio de Berg

Fonte: Dados da pesquisa.

Nota: Escore de corte $=45$ (para risco aumentado de quedas); $0 \mathrm{D}=$ 102; $p<0,0001$. 


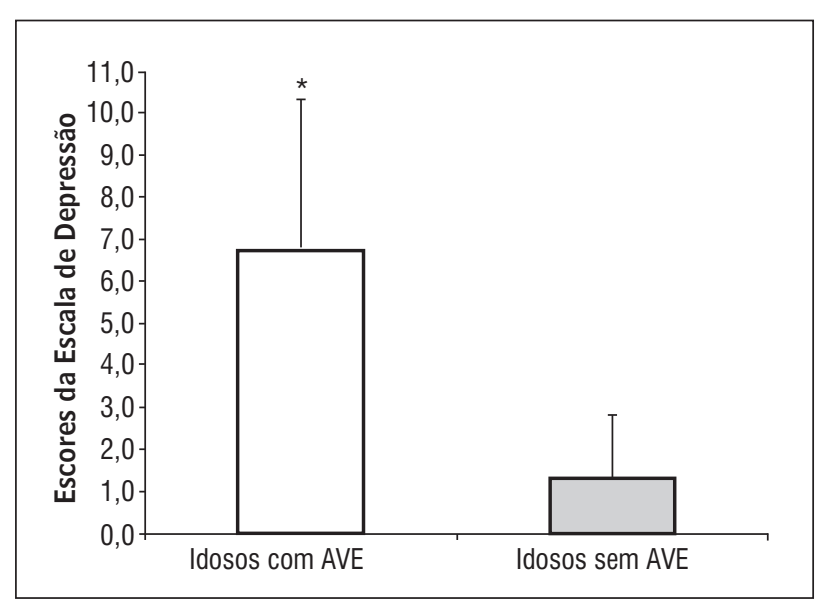

Gráfico 2 - Média da pontuação da Escala de Depressão para idosos com e sem AVE

Legenda: ${ }^{*}=p<0,0001$.

Fonte: Dados da pesquisa.

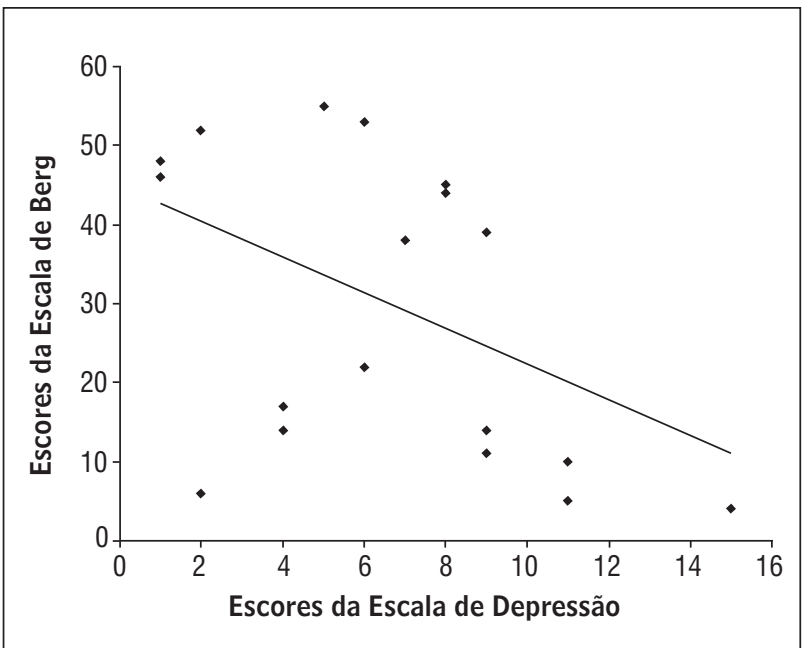

Gráfico 3 - Correlação entre escores da Escala de Depressão e escores da Escala de Berg para idosos com AVE

Fonte: Dados da pesquisa.

Nota: $r=-0,55 ; p=0,01$.

\section{Discussão}

Segundo a literatura, a prevalência de depressão em pacientes pós-AVE pode variar de $23 \%$ a $60 \%$ (19, $11,20)$. Em nosso estudo, 12 participantes $(63,1 \%)$ apresentaram depressão - de acordo com a GDS; resultado pouco superior, portanto, ao que aponta a literatura. Desses pacientes, nove apresentaram depressão moderada e três, depressão grave. A presença de depressão pós-AVE está associada a prejuízo cognitivo, mortalidade aumentada, risco de quedas e pior resultado da reabilitação (21). Nenhum participante do grupo sem AVE apresentou depressão. Esses resultados indicam a necessidade de se avaliar o quadro depressivo em pacientes com sequelas de Acidente Vascular Encefálico.

O impacto psicológico gerado pelas limitações impostas após um AVE poderia explicar o surgimento do quadro depressivo. Apesar disso, parece haver uma questão orgânica relacionada à depressão pós-AVE. Folstein, Maibeger e McHugh (22) relataram uma maior prevalência da depressão pós-AVE quando comparada com pacientes ortopédicos com limitação física semelhante.

A depressão pós-AVE pode trazer comprometimento para a evolução do paciente. Embora em uma unidade de reabilitação os pacientes com depressão pós-AVE possam apresentar recuperação semelhante àqueles não deprimidos, com a alta, e sem a assistência, os deprimidos tendem a apresentar significativa queda da recuperação funcional em comparação com os não deprimidos (23).

Nossos dados mostraram que idosos com AVE têm maior risco de sofrer quedas. É preciso ressaltar que o risco aumentado de quedas não está presente somente na fase aguda do AVE, mas permanece mesmo na fase crônica da doença. Da mesma forma, Meneghetti et al. (23), Yiu et al. (24) e Schmid et al (25) encontraram baixos escores na avaliação do equilíbrio de pessoas com AVE.

Uma explicação para o baixo escore médio da EEB para os pacientes com AVE é que a fraqueza muscular e a espasticidade dificultam o recrutamento coordenado de fibras e a força suficiente para a execução de uma tarefa funcional. A inapropriada resposta muscular e movimentos compensatórios geram um equilíbrio funcional deficiente no paciente, dificultando o controle e a execução da marcha (26). BERG et al. (18), afirmaram que pontuações inferiores a 45 na EEB indicam que um indivíduo está com algum impedimento motor, com um aumento do risco para quedas. No nosso estudo, 14 idosos $(73,6 \%)$ com AVE apresentaram escores inferiores a 45 pontos. Clinicamente, nossos resultados indicam que é necessário iniciar o tratamento de reabilitação precocemente e prevenir as quedas, uma vez que podem trazer sérias consequências (27) como a fratura do colo de fêmur, por exemplo.

A literatura relata relação entre os fatores depressão e equilíbrio $(9,28)$. Nosso estudo mostrou que quanto maiores os escores da GDS, menores os valores obtidos na EEB ( $r=-0,55 ; p=0,01)$. Essa relação pode 
estar relacionada a perda precoce da independência funcional, descondicionamento físico, redução da velocidade da marcha, perda de força muscular por imobilidade e letargia, comuns à depressão (29). 0 valor encontrado da correlação foi moderado $(-0,55)$, pois alguns pacientes com AVE, apesar de baixos escores na EEB, apresentaram valores baixos também na GDS, indicando ausência de depressão. Isso é comum, pois como discutido acima, nem todos os pacientes com AVE desenvolvem um quadro de depressão.

Prata et al. (30) afirmam que há necessidade de mais estudos envolvendo os temas depressão e quedas, além de maior atenção das autoridades, pois são complicações que interferem na qualidade de vida relacionada à saúde.

\section{Conclusão}

Os resultados deste estudo indicam a alta prevalência de depressão em pacientes com AVE crônico e que esse quadro pode levar a inaptidões físicas, aumentando o risco de quedas. Além disso, verificou-se também que o equilíbrio em pacientes com AVE é prejudicado. Por isso, sugere-se que pacientes com AVE crônico sejam constantemente avaliados em relação à depressão e risco de quedas.

\section{Referências}

1. Hazzard W, Ettinger W Jr. Aging and atherosclerosis: changing considerations in cardiovascular disease prevention as the barrier to immortality is approached in old age. Am J Geriatr Cardiol. 1995;4(4):16-36.

2. Sacco R, Adams R, Albers G, Alberts M, Benavente O, Furie K, et al. Guidelines for prevention of stroke in patients with ischemic stroke or transient ischemic attack: a statement for healthcare professionals from the American Heart Association/ American Stroke Association Council on Stroke: co-sponsored by the Council on Cardiovascular Radiology and Intervention: the American Academy of Neurology affirms the value of this guideline. Stroke 2006;37(2):577-617. doi:10.1161/01.STR.0000199147.30016.74.

3. Sanossian N, Ovbiagele B. Prevention and management of stroke in very elderly patients. Lancet Neurol 2009;8(11):1031-41. doi:10.1016/ S1474-4422(09)70259-5.
4. Sacco RL. Risk factors, outcomes, and stroke subtypes for ischemic stroke. Neurology 1997;49:39-44. doi:10.1212/WNL.49.5_Suppl_4.S39.

5. Poynter B, Shuman M, Diaz-Granados N, Kapral M, Grace S, Stewart D. Sex differences in the prevalence of post-stroke depression: a systematic review. Psychosomatics 2009;50(6):563-9. doi:10.1016/ S0033-3182(09)70857-6.

6. Langhorne P, Stott DJ, Robertson L, MacDonald J, Jones L, McAlpine C, et al. Medical complications after stroke: a multicenter study. Stroke 2000;31:1223-29. doi:10.1161/01.STR.31.6.1223.

7. Nyberg L, Gustafson Y. Patient falls in stroke rehabilitation. A challenge to rehabilitation strategies. Stroke. 1995;26:838-42. doi:10.1161/01.STR.26.5.838.

8. Watanabe Y. Fear of falling among stroke survivors after discharge from in patient rehabilitation. Int J Rehabil Res. 2005;28:149-52. doi:10.1097/ 00004356-200506000-00008.

9. Forster A, Young J. Incidence and consequences of falls due to stroke: a systematic inquiry. BMJ. 1995;311:8386. doi:10.1136/bmj.311.6997.83.

10. Robinson RG. Neuropsychiatric consequences of stroke. Annu Ver Med. 1997;48(1):217-9. doi:10.1146/annurev.med.48.1.217.

11. Hackett ML, Yapa C, Parag V, Anderson CS: frequency of depression after stroke. Stroke 2005, 36:1330-40. doi:10.1161/01.STR.0000165928.19135.35.

12. Ensinck $\mathrm{K}$, Schuurman $\mathrm{A}$, van den Akker $\mathrm{M}$, et al. Is there an increased risk of dying after depression? Am J Epidemiol. 2002;156(11):1043-48. doi:10.1093/aje/ kwf148.

13. Glassman A, Shapiro P. Depression and the course of coronary artery disease. Am J Psychiatry. 1998;155(1):4-11. PMid:9433332.

14. Miyamoto ST, Lombardi L Jr., Berg KO, Ramos LR, Natour J. Brazilian version of the Berg balance scale. Braz J Med Biol Res. 2004;37(9):1411-21. doi:10.1590/ S0100-879X2004000900017.

15. Stevenson TJ. Detecting change in patients with stroke using the Berg Balance Scale. Aust J Physiother. 2001;47:29-38. PMid:11552860. 
16. Oliveira R, Cacho EWA, Borges G. Post-stroke motor and functional evaluations: A clinical correlation using Fugl-Meyer assessment scale, Berg balance scale and Barthel index. Arq Neuropsiquiatr. 2006;64(3-B):73135. doi:10.1590/S0004-282X2006000500006.

17. Almeida OP, Almeida AS. Confiabilidade da versão brasileira da Escala de Depressão em Geriatria (gds) versão reduzida. Arq Neuropsiquiatr. 1999;57(2-B):42126. doi:10.1590/S0004-282X1999000300013.

18. Berg KO, Wood-Dauphinee SL, Williams JI, Maki B. Measuring balance in the elderly: validation of an instrument. Can J Public Health 1992; 83 Suppl 2:S7-11. PMid:1468055.

19. Hackett ML, Anderson CS. Frequency, management, and predictors of abnormal mood after stroke: the Auckland Regional Community Stroke (ARCOS) study, 2002 to 2003. Stroke 2006;37(8):2123-28. doi:10.1161/01.STR.0000231387.58943.1f.

20. Sinyor D, Amato P, Kaloupek DG, Becker R, Goldenberg M, Coopersmith H. Post-stroke depression: relationships to functional impairment, coping strategies, and rehabilitation outcome. Stroke. 1986;17(6):110207. doi:10.1161/01.STR.17.6.1102.

21. Paolucci S. Epidemiology and treatment of post-stroke depression. Neuropsychiatr Dis Treat. 2008;4:145-54. doi:10.2147/NDT.S2017.

22. Folstein MF, Maibeger R, McHugh PR. Mood disorder as a specific complication of stroke. J Neurol Nerosurgery Psychiat. 1977; 40:1018-20. doi:10.1136/ jnnp.40.10.1018.

23. Meneghetti CHZ, Delgado GM, Pinto FD, Canonici AP, Gaino MRC. Equilíbrio em indivíduos com Acidente Vascular Encefálico: Clínica Escola de Fisioterapia da Uniararas. Rev Neurocienc 2009,17(1):14-18.
24. Yiu J, Miller WC, Eng JJ, Liu Y. Longitudinal analysis of balance confidence in individuals with stroke using a multilevel model for change. Neurohabil Neural Repair. 2012; 26 (8)

25. Schmid AA, Van Puymbroeck M, Altenburger PA, Dierks TA, Miller KK, Damush TM, et al. Balance and balance self-efficacy are associated with activity and participation after stroke: a cross-sectional study in people with chronic stroke. Arch Phys Med Rehabil. 2012;93(6):1101-7. doi:10.1016/j.apmr.2012.01.020.

26. Chern J, Yang S, Wu C. Whole-body reaching as a measure of dynamic balance in patients with stroke. Am J Phys Med Rehabil. 2006; 85:201-08. doi:10.1097/01. phm.0000200357.98448.d7.

27. 27.Batchelor FA, Hill KD, Mackintosh SF, Said CM, Whitehead $\mathrm{CH}$. The FLASSH study: protocol for a randomised controlled trial evaluating falls prevention after stroke and two sub-studies. BMC Neurology 2009,9:14. doi:10.1186/1471-2377-9-14.

28. Srivastava A, Taly AB, Gupta A, Murali T. Post-stroke depression: prevalence and relationship with disability in chronic stroke survivors. Ann Neurol indiana Acad. 2010;13 (2):123-7. doi:10.4103/0972-2327.64643.

29. Rao SS. Prevention of falls in older patients. Am Fam Physician 2005;72:81-88. PMid:16035686.

30. Prata HL, Alves ED Jr., Paula FL, Ferreira SM. Envelhecimento, depressão e quedas: um estudo com os participantes do Projeto Prev-Quedas. Fisioter Mov. 2011;24(3):437-43.
Recebido: 22/08/2012

Received: 08/22/2012

Aprovado: 08/04/2013 Approved: 04/08/2013 